\section{Un escenario para el aprendizaje a través de proyectos de diseño en contextos sociales: Laboratorio Estratégico de Diseño Integral (LEDI)}

\section{Resumen}

En este artículo se documentan algunas acciones inherentes a la enseñanza del diseño en el marco de una experiencia educativa, analizada desde la práctica proyectual en asignaturas del pregrado de Diseño Visual de la Universidad de Caldas. Se expone la experiencia docente resultante de implementar proyectos de aula, que durante 10 años (2008-2018) de trabajo ha vinculado comunidades y proyectos de ciudad con el pregrado. Se destaca el valor que cobra el contacto con problemas reales en la formación integral de los diseñadores, dando la posibilidad en algunas asignaturas de proponer y planificar acciones didácticas que conduzcan a la mejora de la experiencia de la práctica proyectual. Surge aquí la necesidad de profundizar en la planeación pedagógica, para detectar las características que las acciones didácticas deben presentar y así entender cómo abordarlas desde el diseño, mediante una observación mediada por acercamientos metodológicos cualitativos. El artículo reporta cómo algunos docentes de este pregrado han diseñado contenidos de asignaturas y propuesto escenarios idóneos para la experimentación, dándole espacio al desarrollo de iniciativas como el LEDI (Laboratorio Estratégico de Diseño Integral), que complementa el escenario académico y enfatiza en una visión integral del diseño.

En esta reflexión sobre la práctica docente se plantea una documentación de algunos procesos y proyectos, de los cuales surge el interés de proponer la construcción consciente y contextualizada de los cursos bajo la mirada disciplinar de la educación debido a la necesidad de identificar cómo se orientan los contenidos, tanto teóricos como prácticos de algunas asignaturas de los programas de diseño y sumar a la estrategia educativa la posibilidad de acceder a los espacios profesionales que pueden gestionar los profesores a los cuales pueden acceder los estudiantes desde la universidad y sacar el mejor provecho de la experiencia; así mismo, reflejar la sintonía de las soluciones plantadas desde la academia con las necesidades detectadas del contexto donde influye el proceso de educación, en nuestro caso el pregrado de Diseño Visual, y poner en evidencia la importancia de comprender los espacios sociales donde actúan los diseñadores en formación y acercarlos a estos.
Juliana Castaño Zapata Candidata a doctor en Diseño y Creación.

Profesora asistente, Universidad de Caldas, Manizales, Colombia.

Correo electrónico:

juliana.castano@ucaldas.edu.co

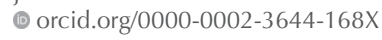
Google Scholar

\section{Óscar Eugenio}

Tamayo Alzate

Postdoctorado en Narrativa y Ciencia.

Profesor titular, Universidad de Caldas y Universidad Autónoma de Manizales, Manizales, Colombia. Correo electrónico:

oscar.tamayo@ucaldas.edu.co

๑ orcid.org/0000-0002-6080-8496

\section{Google Scholar}

Walter Castañeda Marulanda Doctor en Diseño y Creación. Profesor titular, Universidad de Caldas, Manizales, Colombia.

Correo electrónico:

walter.castaneda@ucaldas.edu.co ๑ orcid.org/0000-0002-1466-0910 Google Scholar

Recibido: abril 13 de 2021 Aprobado: junio 24 de 2021

Palabras clave: diseño integral, enseñanza, laboratorio, práctica pedagógica, proyecto de diseño. 


\section{A setting for learning through design projects in social contexts: Laboratorio Estratégico de Diseño Integral, LEDI (Strategic Laboratory of Comprehensive Design)}

\begin{abstract}
Some actions, inherent to the teaching of design within the framework of an educational experience analyzed from the project practice in subjects of the undergraduate Visual Design Program at Universidad de Caldas, are documented in this article. The teaching experience resulting from implementing classroom projects is exposed, an experience that, during 10 years (2008-2018) of work, has linked communities and city projects with the undergraduate Program. The value of contact with real problems in the comprehensive training of designers is highlighted, giving the possibility of proposing and planning didactic actions that lead to the improvement of the experience of project practice in some subject areas. Here arises the need to deepen the pedagogical planning to detect the characteristics that the didactic actions must present and thus to understand how to approach them from design through an observation mediated by qualitative methodological approaches. The article reports how some professors of this undergraduate Program have designed subject content and have proposed suitable scenarios for experimentation, giving space to the development of initiatives such as LEDI Laboratorio Estratégico de Diseño Integral (Strategic Integral Design Laboratory), which complements the academic scenario and emphasizes a comprehensive vision of design.
\end{abstract}

Documentation of some processes and projects arises in this reflection on the teaching practice, from which the interest arises to propose the conscious and contextualized construction of the courses under the disciplinary perspective of education due to the need to identify how contents, both theoretical and practical, of some subjects of the Design Programs are oriented and add to the educational strategy the possibility of accessing professional spaces that can be managed by professors, and that students can access from the university and get the best out of this experience, as well as reflecting the harmony of the solutions planted from the academy with the detected needs of the context where the education process influences, in this case, the undergraduate Program in Visual Design and highlights the importance of understanding the social spaces where in-training designers act and bring them closer to them.
Key words:

comprehensive design, teaching, laboratory, pedagogical practice, design project. 


\section{Introducción}

Para comprender el alcance de la reflexión que aquí se presenta es preciso dar sentido a las prácticas pedagógicas universitarias, especialmente a aquellas relacionadas con la enseñanza, en las que el docente planifica un conjunto de estrategias y acciones para la formación integral de sus estudiantes (Loaiza, 2019), donde su implementación "permitirá al educando desarrollar habilidades cognitivas, emocionales, físicas y psicológicas, pero, además, contribuirá a su formación como seres humanos libres, pensantes, autónomos, autorrealizados y éticos" (p. 51).

De esta manera, presentamos en este artículo un escenario universitario donde se desarrolla una experiencia docente en el campo del diseño. Al documentar el ejercicio se presenta una práctica alternativa y motivante para los profesores de la carrera de Diseño Visual de la Universidad de Caldas, todo dentro de un marco de políticas institucionales que propician espacios educativos diversos para los estudiantes y retadores para los docentes.

En un inicio, se trató de vincular un proyecto de enseñanza-aprendizaje del diseño con espacios de proyección profesional, lo que, si bien ha sido una práctica pedagógica usada frecuentemente en el aula de diseño, en el caso presentado evoluciona a la propuesta de un Laboratorio Estratégico de Diseño Integral (LEDI) (Figura 1). 


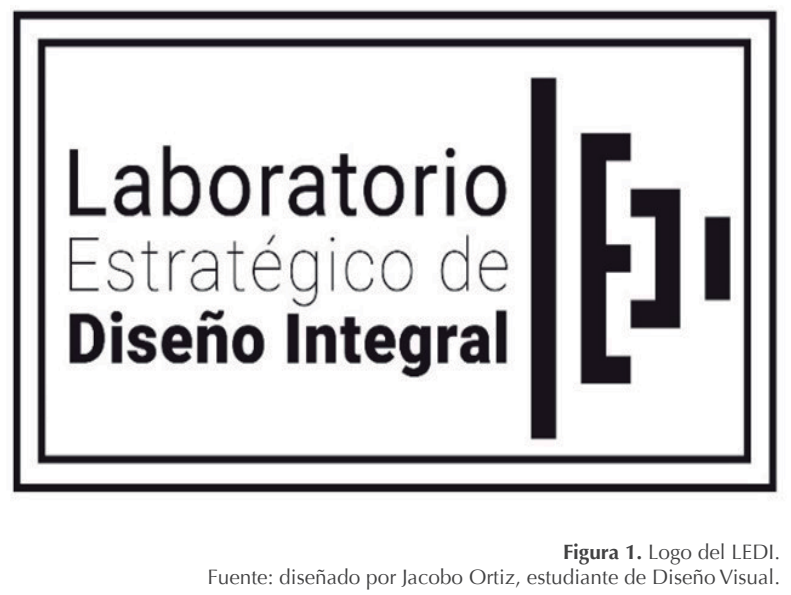

Así, el laboratorio surge en el marco de las políticas institucionales plasmadas en el Plan de Acción Institucional de la Universidad de Caldas, denominado "Universidad y Pensamiento Crítico" (Londoño, 2014), donde se encontraron tres ejes fundamentales como rutas para el programa de gobierno: Humanismo como eje transformador, Conocimiento útil a la sociedad y Excelencia académica (PAI, 2014-2018).

Para esto, la rectoría del momento partió del saber multidisciplinar como premisa articuladora de este plan de acción, lo que permitió el surgimiento del LEDI, con el fin de generar proyectos desde las unidades académicas de Diseño Visual, donde diferentes disciplinas encuentran en el diseño un área del saber que gestiona, articula, modela e implementa procesos para desarrollar acciones proyectuales desde la integración no solo de conocimientos sino, sobre todo, de experiencias de los diferentes sujetos que deseen participar. 
Es así como a partir de proyectos en el aula e iniciativas de integración con el contexto práctico, se abre un espacio para la realización de trabajos profesionales, por ejemplo, para la producción audiovisual donde los estudiantes se conectan con procesos de ciudad, como es el caso de Manizales Grita Rock, festival de música que sumado al interés en especial desde asignaturas como Diseño Integral y otras como Laboratorio de Diseño Audiovisual presentes en el pensum del programa de Diseño Visual, se ha presentado la oportunidad de aportar con la construcción de proyectos culturales como el Visual Rock y se detecta a su vez la necesidad de crear un escenario de experimentación docente, que logra articularse con la investigación educativa y de diseño. Es así como la asignatura le da vida al laboratorio, a su vez, el laboratorio dinamiza los procesos de aprendizaje y proyectos de diseño de las asignaturas, y el docente articula vías de enseñanza con los recursos disciplinares y profesionales.

El aspecto que caracterizaría este Laboratorio Estratégico es el Diseño Integral, que se entiende desde la concepción del potencial que tiene el diseño, como disciplina, de incidir en el contexto, ya que "la visión integral del diseño establece la articulación de las formas, mediante los procesos del diseño, superando los procedimientos particulares y ampliando su perspectiva hacia la estrategia y la concepción misma del diseño" (Plan Institucional de Actividad Académica, 2015).

En consecuencia, la motivación más importante de este proyecto del LEDI es la construcción de un espacio de exploración educativa docente, que busca responder a las expectativas de la comunidad con respecto a lo que el diseño puede hacer por sus necesidades. Inicialmente, desde el laboratorio se contactan proyectos internos de la Universidad y fundaciones sin ánimo de lucro de la región, porque su función social facilita el planteamiento de objetivos sin acudir a convenios o actividades contractuales. 
Para cumplir con los propósitos formativos y sociales de las entidades contactadas, se realizan diagnósticos de lo que el diseño puede hacer por sus propuestas, desde las tipologías que aborda el diseño visual, como lo son el diseño de imagen gráfica, audiovisual, de interfaces y ambiental, dentro del marco del diseño integral, planteando la proyección de una idea como una posibilidad de modelar procesos, encontrar soluciones y proponer acciones.

Para la enseñanza del diseño es necesario reconocer las diferentes dimensiones de la formación que los estudiantes deben tener, tanto para los contenidos de la disciplina como los de la profesión. En las carreras de diseño se busca hacer énfasis en las bases técnicas, teóricas y procedimentales para la práctica proyectual y comprender la relación de esta con el contexto social. En concordancia con lo anterior, uno de los objetivos de cualquier buen profesional de la educación consiste en ser cada vez más competente en su oficio (Zabala, 2000, p. 11), en este caso la motivación que cualquier docente de diseño debería tener es proponer acciones didácticas coherentes y consistentes con la naturaleza de la disciplina, que permitan realizar en el aula intervenciones que representen para el estudiante un aprendizaje profundo.

En este sentido, el aprendizaje profundo está ligado a la experiencia de cada estudiante, "construyendo por él mismo el conocimiento, no aprende de la experiencia del otro, se aprende construyendo las propias experiencias y es a partir de esta experiencia el significado que le damos al mundo" (Ortega-Díaz y Hernández-Pérez, 2015, p. 215). En consecuencia, vale la pena analizar desde la enseñanza del diseño cuáles han sido los escenarios y los mecanismos que han proporcionado vías para que los estudiantes logren este tipo de aprendizaje. Como lo presentan López y Tamayo (2012), al justificar la pertinencia de los laboratorios como complemento en la formación universitaria: 
(...) en el caso de los procesos educativos, la actividad experimental hace mucho más que apoyar las clases teóricas de cualquier área del conocimiento; su papel es importante en cuanto despierta y desarrolla la curiosidad de los estudiantes, ayudándolos a resolver problemas, a explicar y comprender los fenómenos con los cuales interactúan en su cotidianidad. (p. 148)

Así, se han utilizado proyectos de diseño para el aprendizaje práctico en algunas asignaturas, a través de actividades proyectuales que corresponden a secuencias didácticas propias de los laboratorios, talleres y experiencias de formación de algunas asignaturas en el pregrado de Diseño Visual de la Universidad de Caldas. Entendiendo por secuencia didáctica las acciones educativas que permiten planificar el desarrollo de las clases y las formas en las cuales se utilizarán recursos que contribuyan al aprendizaje de los estudiantes (Tobón et al., 2010).

Explorar esta experiencia desde la pedagogía ha perfilado la investigación doctoral en curso, "El proyecto de diseño como recurso didáctico de dominio específico que contribuye a la formación de pensamiento crítico en diseño", desde la cual se genera el interrogante sobre qué acciones didácticas influyen en la construcción de los proyectos en el aula de diseño que, para el caso de este artículo, expone la experiencia acumulada a través de diez años (2008-2018) en docencia en el programa de Diseño Visual.

Se busca, entonces, comprender qué tipo de conocimientos además de los técnicos, teóricos y procedimentales se alcanzan gracias a los proyectos para la enseñanza del diseño, pues al tener un escenario práctico, profesional y real como lo es el LEDI en donde el componente del impacto social es determinante, se pretende que la experiencia motive en los estudiantes unas reflexiones profundas de su quehacer. 


\section{Método}

Los laboratorios son escenarios heredados de las ciencias naturales, que se han implementado en ambientes académicos. En el caso de una disciplina proyectual y creativa como lo es el diseño, el espacio del laboratorio surge como respuesta del proceso de formación de los estudiantes en función del desarrollo de condiciones en las que se puedan experimentar y explorar situaciones cotidianas, relacionadas con el ejercicio, en este caso, del diseño visual. Se procura, entonces, aportar a la formación de profesionales integrales mediante prácticas que ofrecen experiencias de naturaleza técnica difíciles de hallar en otros escenarios de aula, como lo son los talleres o actividades en las que se simulan problemas.

La tarea del docente, al estar mediada por la respuesta de sus desafíos en el aula y en el contexto, lo invita a una constante renovación, "se requiere para ello un cambio en las formas aceptadas de la didáctica y la enseñanza de las disciplinas o áreas del conocimiento que se imparten en la educación superior" (Díaz et al., 2021, p. 169).

En tal sentido, se considera que los laboratorios fungen como escenarios de orden experimental que siguen procedimientos sistemáticos, ordenados, transparentes, transferibles y procedentes, que en cierto sentido deben ser ajustados para los procesos generativos e iterativos que se presentan en el diseño. Por tal motivo, se considera interesante analizar el caso del LEDI, como un espacio en el que si bien se incluyen metodologías generales del diseño como las de Gugelot (1963, como se cita en Rodríguez 2006), Alexander (1980) o Cross (2012), entre otros, se buscan otras según los proyectos que abordan los estudiantes, que enmarca a este laboratorio como un lugar particular de prácticas y experiencias pedagógicas. 
Por lo anterior, se aborda este problema de reconocimiento del LEDI como un espacio particular de exploración docente, a partir de un paradigma cualitativo (Creswell, 1998; Hernández et al., 2014), que se fundamenta en la observación y descripción de aspectos actitudinales de los participantes, con el fin de aproximarse a comprender cómo los estudiantes afrontan las distintas etapas del proceso proyectual, identifican los problemas, interactúan con los clientes y planifican las soluciones contextualizando el fenómeno para su proyecto.

Se considera el LEDI como un escenario para la formación de profesionales en diseño, escenario en el cual los estudiantes aprenden a resolver problemas de la vida profesional. A partir de su formación en Diseño Visual, con habilidades para comprender los ámbitos de la imagen, los estudiantes realizan acciones específicas, propuestas por los docentes, de las asignaturas, en función del logro de aprendizajes profundos de los temas estudiados. La plataforma metodológica que sirve como soporte reconoce el valor de los estudios de naturaleza cualitativa, fundamentales en la comprensión detallada de los procesos de pensamiento y acción característicos para la formación de profesionales en diseño.

El LEDI se propone como un espacio para la experimentación que, mediante la concepción del Diseño Integral, acerque a los estudiantes para que logren perfeccionar sus habilidades profesionales y sociales, por tanto, la pretensión es la de interpretar la adecuación de los estudiantes, para afrontar problemas de 'la vida real', en condiciones similares a las que tendrán en su desempeño profesional. De acuerdo con esos principios, se analiza y describe la experiencia alcanzada en referencia a 54 productos vinculados a prácticas educativas desarrolladas a lo largo de 10 años de experiencia docente en asignaturas de Diseño Visual, en las que se describe el ejercicio profesional lo más inmerso en la práctica que permite vislumbrar el objetivo de la asignatura de diseño 
integral se cumple de manera satisfactoria según lo esperado y que atiende a la consolidación de las habilidades profesionales y sociales del diseñador visual.

\section{Resultados - reflexión}

En el presente artículo se reportan procesos de experiencia de aula, y se plantea una reflexión para mostrar el impacto que han tenido algunas acciones educativas, en la articulación de varias asignaturas con iniciativas culturales de la región e instituciones sin ánimo de lucro. Desde este lugar, se plantea un escenario académico alternativo para desarrollar proyectos: el Laboratorio Estratégico de Diseño Integral (LEDI), que ha permitido a los estudiantes consolidar su aprendizaje en escenarios profesionales, donde fuera de la práctica académica se propone una experiencia con el medio real, tal como se presenta en la Tabla 1:

Tabla. Síntesis de las entidades involucradas con LEDI

\begin{tabular}{llll}
\hline Año & Proyecto & Entidad & Impacto \\
\hline $2009-2014$ & Visual Rock & Bandas Manizales Grita Rock & $\begin{array}{l}38 \text { videos con 446.998 visitas totales a marzo } \\
\text { de 2021. }\end{array}$ \\
\hline 2015 & Diseño Integral & Fundación Nutrir & $\begin{array}{l}\text { Nueva propuesta de diseño de la página web } \\
\text { y diagnóstico de diseño ambiental en sus } \\
\text { comedores infantiles. Se entregaron artes finales } \\
\text { y propuestas gráficas. }\end{array}$ \\
\hline 2015 & Diseño Integral & Vereda El Águila & $\begin{array}{l}\text { Trabajo con la comunidad del sector para la } \\
\text { apropiación de su espacio de integración y } \\
\text { encuentro. Se entrega señalética y marca. }\end{array}$ \\
\hline 2015 & Diseño Integral & Escuela Londoño Jaramillo & $\begin{array}{l}\text { Adecuación de una ludoteca. Talleres con los } \\
\text { niños. }\end{array}$ \\
\hline
\end{tabular}




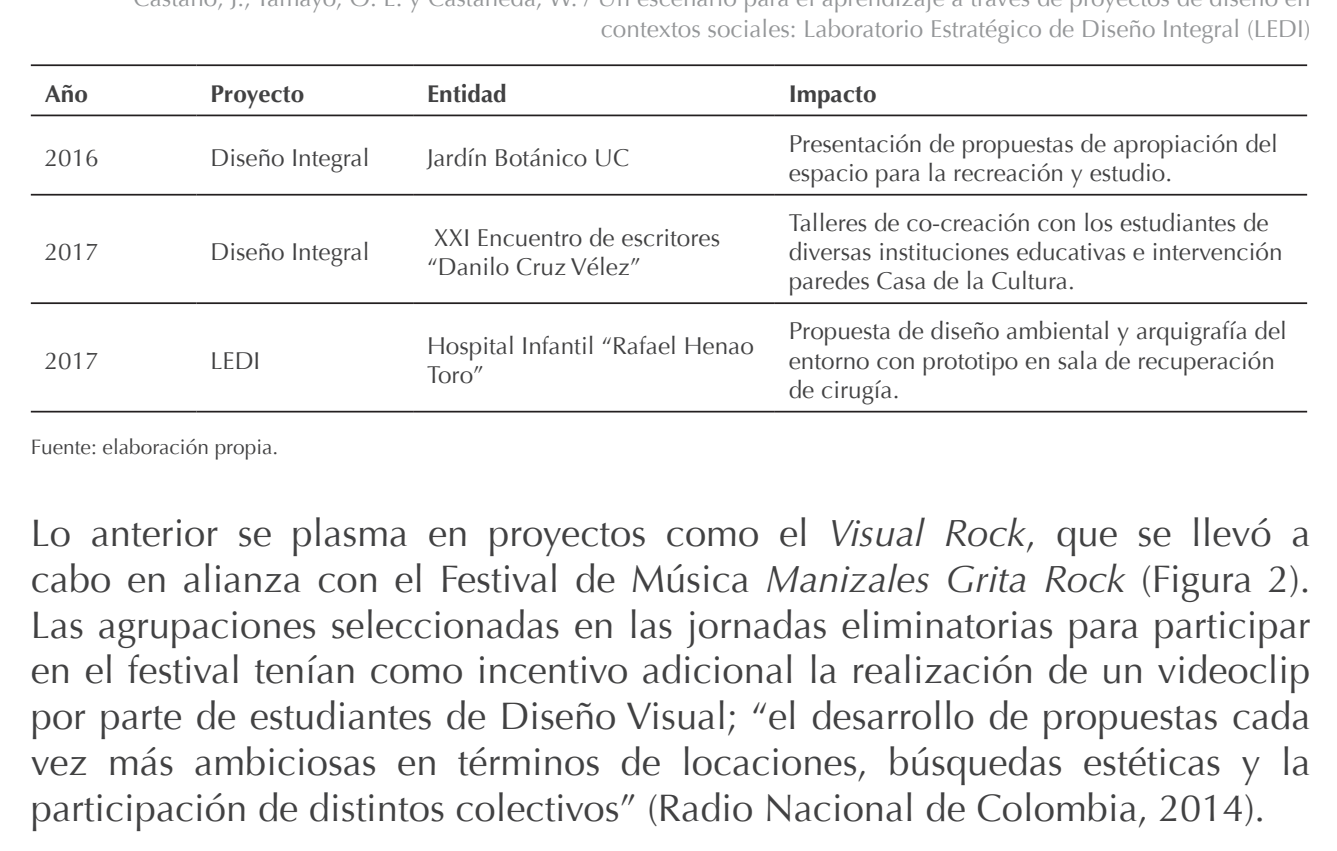

145 


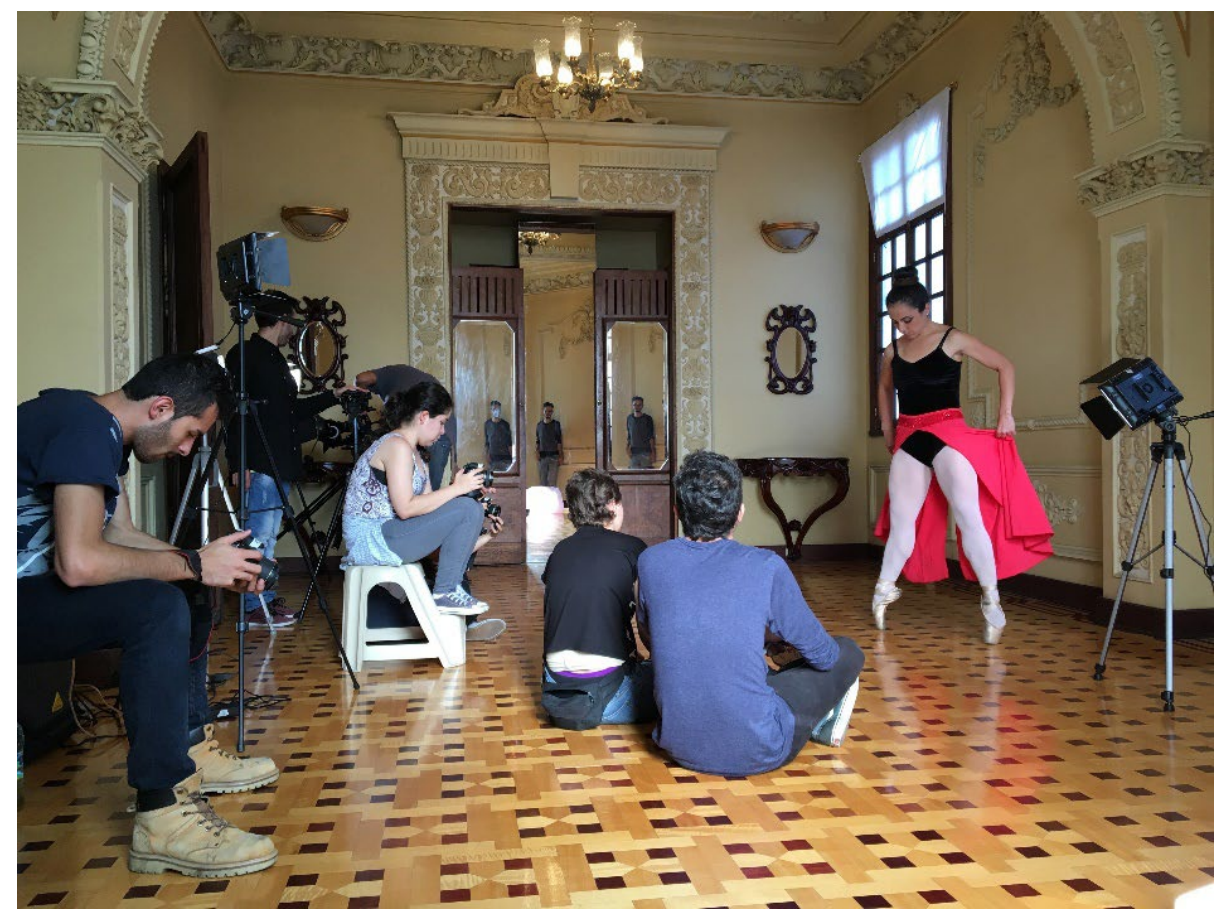

Figura 2. Grabación del video "El sueño", banda Fúnebre, para Manizales Grita Rock. Locación: Gobernación de Caldas, 2016. Fuente: Propiedad de los autores.

En el caso de la colaboración con la Fundación Nutrir (2021), ubicada en Manizales (Colombia) y creada en 1986 con el fin mejorar las condiciones nutricionales y la calidad de vida de la población infantil y las madres gestantes y lactantes en condición de vulnerabilidad del departamento de Caldas, en este proyecto los estudiantes realizaron trabajo de campo visitando los comedores comunitarios autorizados por los programas de la fundación, tuvieron reuniones con la encargada de las comunicaciones y con la directora 
de la fundación para conocer a profundidad los deseos y necesidades, y plantearon varias estrategias en diferentes grupos. Unos se encargaron de la restructuración de la página web, otros de la ambientación de los comedores como espacios educativos extracurriculares y, por último, otros presentaron propuestas de souvenirs y productos de diseño que sirvieran para apoyar las actividades de la fundación y recoger dinero adicional.

Otro proceso de inmersión en el contexto a través de un proyecto en el aula se dio en la escuela pública rural Londoño Jaramillo, ubicada la vereda Playa Larga (Villamaría, Caldas). Es el segundo centro educativo caldense más cercano al Volcán Nevado del Ruiz, donde se trabaja bajo el modelo de Escuela Nueva y se representa un reto el acceso a la educación de la población infantil (Figura 3). En este lugar, los estudiantes de Diseño Visual que cursaban la asignatura de Diseño Integral se acercaron a la comunidad identificando las necesidades evidentes en su aula de clase, y como acción de diseño se realizó la construcción de una ludoteca y la organización del espacio de trabajo para mejorar la distribución espacial del salón, también se realizó una dotación de material didáctico y la visita para compartir y conocer a estos niños que enfrentan desafíos para acceder a la educación. 


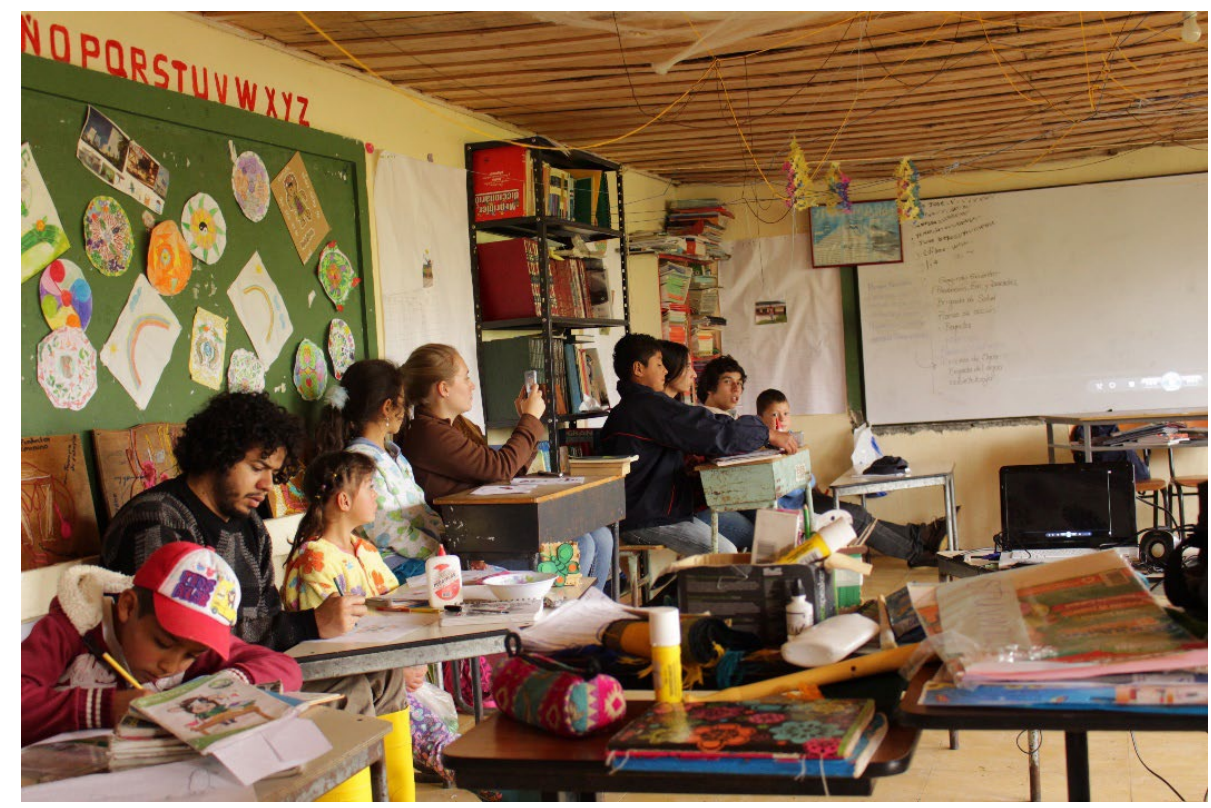

Figura 3. Visita a Escuela Londoño Jaramillo Villamaría, Caldas, 2014. Fuente. Propiedad de los autores.

La última intervención se realizó en 2017 con acciones en el Hospital Infantil, con la propuesta de señalética y arquigrafía del entorno hospitalario para niños. Aquí los estudiantes visitaron el hospital en varias ocasiones y sostuvieron reuniones con la trabajadora social, el gerente y la encargada de la salud ocupacional. Se detectaron posibilidades de adecuación señalética y pediatrización del espacio (Figura 4), se estudió a profundidad el reto de introducir imágenes dentro de un centro de atención infantil, se escucharon las opiniones de médicos y enfermeras, se entregó como donación la aplicación de la propuesta para la sala de ingreso a cirugía y se dio el manual de aplicación de la propuesta visual y señalética (Figura 4). 
Como con los anteriores, se han realizado acompañamientos a proyectos de la Vicerrectoría de Proyección como el del barrio Villa Hermosa y Aguas de Manizales, en la exploración de alternativas para embellecer el barrio; el trabajo en la vereda el Águila km 5 vía Neira con la comunidad del sector y la Junta de Acción Comunal; apoyando el semillero dancístico de la universidad; asistiendo a trabajo en la comunidad del municipio de Filadelfia (Caldas) y apoyando actividades de la Casa de la Cultura y el encuentro de escritores.

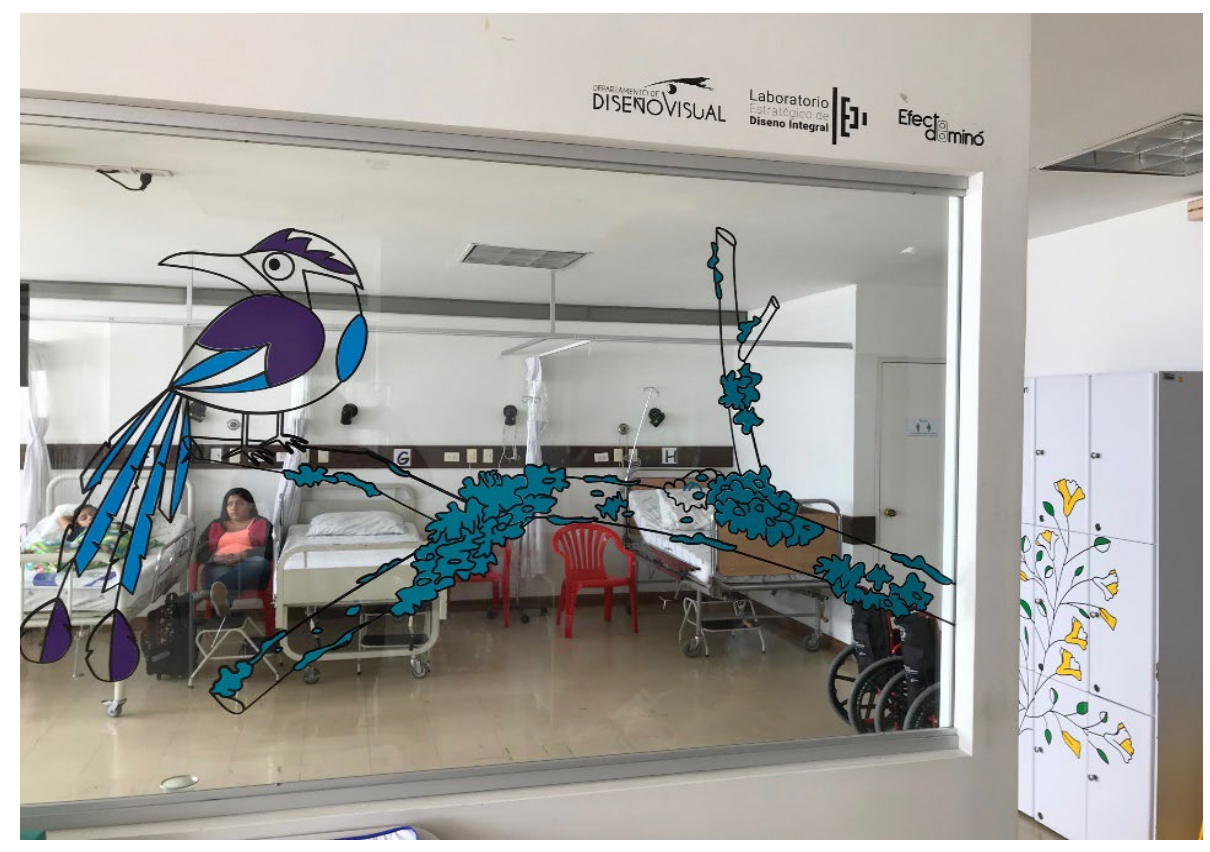

Figura 4. Sala de ingreso a cirugía, Hospital Infantil "Rafael Henao Toro", Manizales, 2017 Fuente: Propiedad de los autores. 
Todas estas experiencias han permitido a los estudiantes apropiarse del LEDI para realizar acciones que les permiten conocer el contexto, escuchar a las personas de diferentes comunidades y comprometerse con el factor social que involucra su acción como diseñadores en el medio.

Rodríguez (2011) pone en el escenario la discusión si en las instituciones donde se enseña actualmente el diseño se tienen en cuenta las realidades emergentes de la profesión y los avances de la disciplina. Él propone la urgencia de nuevos modelos de enseñanza donde el enfoque de la disciplina vaya más allá de la creación de objetos, es decir, una conceptualización más amplia del diseño, en donde los diseñadores logren proyectarse como profesionales que resuelven problemas de carácter integral.

La experiencia de realizar proyectos reales dentro de asignaturas del programa de Diseño Visual ha permitido desde la docencia articular procesos de enseñanza donde los estudiantes puedan realizar diagnósticos y, en lo posible, ejecutar acciones que fortalezcan y dinamicen los escenarios de la práctica profesional real. Todo esto con el fin de trasladar lo aprendido durante la carrera a escenarios profesionales, afianzar el aprendizaje profundo y desarrollar un pensamiento crítico en el momento de tener contacto con diversos sectores de la sociedad.

Es oportuno que los docentes saquemos provecho de las alianzas estratégicas con proyectos externos a la Universidad, que amplían la cobertura de la población y evidencian un amplio espectro de proyectos a los cuales se puede llegar desde el quehacer del diseño.

Gracias a este escenario alternativo de docencia y aprendizaje, que busca generar acciones concretas que se dan desde la academia para aportar en contextos sociales, se logra no solo lo que se conoce como apropiación social 
del conocimiento, sino también de investigación, ya que estas intervenciones han trascendido las actividades de enseñanza-aprendizaje hasta el punto de integrarse al semillero de proyección solidaria 'Efecto Dominó'. Todo esto sumado al interés del Departamento de Diseño de la Universidad de Caldas para formalizar estos procesos del aula, como proyectos permanentes en el marco de la proyección universitaria, con lo que se logra fomentar las acciones de los estudiantes en la comunidad local.

Con lo anterior se hace pertinente, entonces, analizar desde el ámbito educativo cómo estas decisiones en relación con la enseñanza del diseño deben ser pensadas por parte de profesores para que tengan mayor impacto en la formación de los estudiantes de diseño visual. Al revisar las asignaturas y los proyectos que han inspirado la reflexión de esta experiencia educativa se reconocen con cierta facilidad los aspectos de diseño que permean la planeación del curso; no tan evidentes son los componentes didácticos esenciales en función del logro de aprendizajes profundos en los estudiantes y, tal vez lo más importante, en relación con el aporte a la formación de profesionales críticos.

Por lo tanto, la primera acción es proponer secuencias didácticas que permitan el tránsito de lo teórico a lo práctico, ya que en la formación en diseño es fundamental integrar los aspectos teóricos desde diferentes posturas conceptuales, ideologías, métodos de diseño y teorías de otras disciplinas, que complementan el campo de acción, con aspectos específicos de la práctica profesional como lo son las habilidades técnicas y tecnológicas que se esperan de los diseñadores. En resonancia con lo anterior, las siguientes son las acciones que se implementaron en el LEDI, como parte de la secuencia didáctica:

1) Conformar un banco de proyectos internos de la Universidad que puedan vincular al diseño como agente dinamizador de los mismos; y externos que permitan generar alianzas estratégicas con fundaciones sin ánimo de lucro, para 
realizar el rastreo de la pertinencia del aporte desde el diseño en los proyectos que estas entidades plantean. Las alianzas se realizan con la participación de los estudiantes de las asignaturas, mediante lluvias de ideas o presentación de casos donde se identifican posibles situaciones para abordar desde el diseño.

2) Luego de identificar cuáles son los proyectos relevantes y significativos para el LEDI, se eligen dos proyectos internos de la universidad y uno externo para desarrollarlos en clase, según el interés de los estudiantes. Con lo anterior, se puede ofertar la asignatura dos veces al año asumiendo así cuatro proyectos internos y dos externos anualmente. Esta selección se hace en consulta con el Departamento de Diseño, donde se presenta ante el colectivo docente si es un proyecto de amplio aliento o se busca el visto bueno del director según la pertinencia de estos y la idoneidad de las alianzas que generen dichos proyectos.

3) Posteriormente, se plantea la conformación de grupos de trabajo de estudiantes para un primer acercamiento a los proyectos, se presenta una socialización de los mismos por parte del coordinador de la iniciativa o el encargado de presentar la problemática desde los proyectos externos, o propios de la Universidad. Con este insumo se aplica inicialmente el proceso de diseño Design Thinking (Brown, 2009, p. 3), que pretende generar ideas innovadoras al centrar su eficiencia en entender y dar solución a las necesidades reales de los usuarios. Para este punto, los estudiantes de la asignatura de Diseño Integral ya han cursado cuatro clases magistrales donde se han debatido temas como el rol del diseñador, el papel del diseño en la sociedad actual, el avance de la disciplina y la profesión, además de conocer a profundidad cómo se implementa el Design Thinking para poder aplicarlo, desde la concepción de ser un proceso o metodología de diseño que proporciona un enfoque basado en soluciones para resolver problemas. 
4) Luego de esto, se fracciona el grupo en colectivos de diseño y se exploran los resultados de la aplicación inicial del Design Thinking, con el fin de iniciar los ejercicios prácticos donde se aborda el tema de los métodos de diseño para ser aplicados en los proyectos de los estudiantes, quienes exploran posibles perspectivas como las de Morris Asimov (1970), Hans Gugelot 1963, como se cita en Rodríguez, 2006) Leonard Bruce Archer (1964), John Christopher Jones (1976), Abraham Moles y Roland Caude (1977), David Lauer (1979), Bruno Munari (1981), Per Mollerup (1987), Norberto Chaves (1988), Elena González Miranda (1994), Bryan Lawson (2005), Jorge Frascara (2006), Curt Cloninger (2007), André Ricard (2008), Gavin Ambrose y Paul Harris (2010), entre otros. Los estudiantes deben considerar cuáles métodos serían los adecuados para proyectos orientados al diseño gráfico, otros al de producto, otros al ámbito empresarial, según las tipologías del diseño que se acomoden al encargo o problema de diseño, y analizar la función de estos métodos y así resolver el asunto de la pertinencia del método en cada proyecto.

Para ilustrar lo anterior, un ejemplo donde el estudiante es quien propone el proyecto, puede tomar la referencia del método propuesto por Gugelot (1963), referenciado por Rodríguez, que fue adoptado por la escuela Ulm, a partir de lo que denominó como "diseño científico. Se caracteriza por la integración del proceso de diseño en el proceso de producción y por la elaboración de una metodología proyectual, es decir, por dotar de carácter metodológico al proceso de creación" (Rodríguez, 2006, p. 30). Su modelo procedimental de diseño consta de las siguientes etapas (Figura 5): 


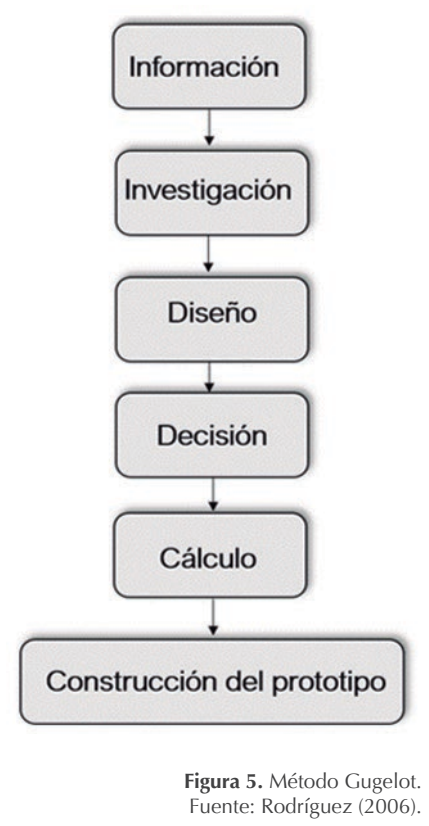

En el caso del trabajo que involucre el encargo de un cliente o comunidad, la metodología de Jorge Frascara (2006) puede ser más útil para los estudiantes.

5) De implementarse el trabajo de diseño con cliente o alianzas con proyectos realizables, se debe formalizar el convenio ante la Universidad y el Departamento de Diseño para garantizar el crédito de los estudiantes participantes y su propiedad intelectual.

6) En los proyectos donde se desarrolla solo un diagnóstico, los estudiantes proponen referentes para las soluciones de diseño sin comprometer su propiedad intelectual. 
7) Se trabajan los proyectos como parte integral del curso permitiendo la evaluación del progreso del estudiante, mediante el acompañamiento y la retroalimentación de cada proceso proyectual.

8) Se exponen las entregas finales ante los clientes, en este caso los delegados de las fundaciones o proyectos, y se realiza una autoevaluación del proceso de aprendizaje y la experiencia práctica.

En la experiencia del LEDI se plantea entonces el aprendizaje basado en proyectos, modalidad tomada desde la educación y que debe ser adaptada y comprendida ante los proyectos de diseño y el uso de métodos de diseño como recursos disciplinares para llevar a cabo proyectos reales, se prueban los métodos y se evidencia el potencial del proyecto de diseño como recurso didáctico propio de la disciplina.

\section{Hallazgos}

En el plan de mejoramiento de Diseño Visual, se plantea la relevancia académica y pertinencia social del programa, escenario en el cual se propuso el Laboratorio Estratégico de Diseño Integral (LEDI), con el fin de fortalecer tanto la cantidad como el tipo de proyectos de carácter social, y así apoyar las funciones de docencia, extensión e investigación de su capacidad docente.

Teniendo como principal referente el alcance y el impacto de proyectos como el Visual Rock y los que gracias a esta experiencia se comenzaron a abordar en la asignatura Diseño Integral, se evoluciona a la propuesta del LEDI como parte de la evolución de la práctica pedagógica docente.

También, se ofrece a los estudiantes la participación en actividades de formación integral, al propiciar proyectos de investigación en semilleros de investigación 
y proyección, que permiten la formalización de algunos proyectos de grado de los estudiantes inscritos bajo la modalidad del LEDI. Este impacto puede contribuir a la meta del Plan de Acción del Departamento de Diseño - PADE, que busca incrementar el número de estudiantes de pregrado de la Facultad que se gradúen bajo la modalidad de semillero y así motivar la formación de nuevos investigadores.

A partir del análisis de la experiencia educativa que ha generado el LEDI, se pueden describir diversas dimensiones que nutren las prácticas pedagógicas del diseño, como lo son:

- Lo Social: El estudiante de diseño se vincula a proyectos que le permiten conocer y detectar necesidades en su entorno cercano a través del trabajo interdisciplinar, al integrarse como sujeto activo de una comunidad, y puede asumir una actitud propositiva frente a los retos que plantea cada uno de los proyectos.

- Lo Cultural: Al encontrarse el LEDI en la Facultad de Artes y Humanidades, en la que se incluyen los programas de Música, Artes Escénicas, Artes Plásticas y Diseño, los estudiantes de diversas áreas pueden articular sus prácticas a través de proyectos multidisciplinarios y con proyectos activos de la Facultad y del Departamento de Diseño que puedan impactar los diferentes escenarios de la ciudad y la región. Esta idea busca la apropiación y activación de los espacios culturales por parte de los estudiantes, ya que gracias a esta iniciativa se pueden plantear diversas intervenciones académicas comprometidas con el contexto social.

- Otro escenario posible donde el LEDI puede intervenir desde la cultura es en el trabajo articulado con el sector artesanal, donde el Departamento de Diseño desde 2009 ha buscado espacios de acción y tiene la meta de 
desarrollar un programa técnico. También desde los programas especiales que tienen la cultura como centro, la incubadora de empresas culturales y el ClusterLab, donde se presentan y desarrollan proyectos multidisciplinarios de la industria cultural y creativa, proyectos de la Facultad de Artes y Humanidades que han tenido vasta acogida con el Ministerio de Cultura y el sector cultural de la región.

- Lo Económico: Como proyecto de proyección universitaria, se puede potenciar el uso de los recursos propios de la institución en la producción de proyectos académicos y profesionales que impacten cultural y socialmente la comunidad universitaria inicialmente. Adicional a esto, al establecer alianzas estratégicas con fundaciones sin ánimo de lucro, la Universidad aporta la capacidad de trabajo para cumplir con su responsabilidad social y visibilizarse a nivel regional, como se ha logrado hasta el momento con proyectos como el Visual Rock, la Fundación Nutrir y el Hospital Infantil, lo que permite aprendizajes en contextos reales para los estudiantes, además de la gestión y ejecución de recursos para los proyectos de diseño propuestos.

- Lo Ambiental: Desde el LEDI una de las preocupaciones es ayudar a los proyectos a identificar qué acciones pueden generar procesos sustentables, además de optimizar diversos tipos de materiales amigables con el ambiente. Desde la educación en diseño urge asumir retos de problemas como el cuidado del agua, la protección de los animales y el cuidado del entorno donde se desarrollan las actividades humanas y los recursos que deben salvaguardarse.

Así, con la iniciativa del LEDI propuesto desde la Proyección Universitaria, se pretende que los diseñadores en formación asuman que son agentes de cambio y tienen la posibilidad de realizar acciones en sus contextos para 
transformar y ayudar a la comunidad. Gracias a la intervención personal dentro de los proyectos y el intercambio de experiencias con otros profesionales, los estudiantes ponen de manifiesto el conocimiento útil a la sociedad desde su proceso de formación, al demostrar la excelencia académica que desde el Departamento de Diseño se les propone, dando la posibilidad de ver cómo sus ideas y conceptos pueden aportar a las diferentes áreas del conocimiento y verlas materializadas en el contexto.

Wenger (1998) describe el aprendizaje desde el concepto de "comunidades de práctica", como algo que no solo se hace individualmente cuando se está en el ambiente educativo, sino también a lo largo de la vida en contacto social con personas que tienen preocupaciones comunes. Estas comunidades de práctica se presentan tanto en grupos de intereses especiales cuyos miembros dedican atención al mismo tema, como también en personas que construyen relaciones significativas a través de las cuales aprenden mutuamente sobre la práctica profesional, al compartir experiencias, formas de pensar sobre el trabajo que realizan y una red de conexiones que los distingue de los demás (Lave y Wenger, 1991; Davis, 2016).

Desde lo académico, con este proyecto del LEDI se pretende afianzar el perfil del diseñador visual desde las comunidades de práctica, al aplicar sus conocimientos en los proyectos que aquí se desarrollen, que le permiten alcanzar habilidades formativas propuestas en el plan de estudios, tal como se proponen desde el programa de Diseño Visual (http://www.disenovisual.com/ diseno-visual/), como se enuncia a continuación:

Mediante las prácticas proyectuales del laboratorio se plantea la forma de generar mensajes visuales acordes a los requerimientos del medio, aplicando los principios del diseño, y a su vez valorar los modos de la comunicación, 
en su forma más contemporánea, y los tipos de la imagen actual, para la formulación de realizaciones de diseño significantes.

Como desafío a los diseñadores en formación frente al uso de los diferentes soportes de la imagen, se les propone desarrollar sistemas articulados de signos visuales, para generar mensajes en los soportes de la imagen fija, elaborar proyectos audiovisuales, evaluando y proponiendo adecuaciones formales con los principios que rigen la construcción de las imágenes audiovisuales en el espacio y su articulación en el tiempo.

A su vez, se invita a realizar proyectos de diseño en las diferentes escalas del espacio tridimensional, que contribuyan a mejorar la comunicación, la interacción, el comportamiento y las relaciones de las personas en su entorno visual ambiental.

Con esto se fortalecen proyectos multidisciplinarios que integren componentes digitales, categorías estéticas y funcionales relacionadas con la creación proyectual, desde una perspectiva crítica y formal. Por lo tanto, con la incursión del laboratorio en las asignaturas de Diseño Visual se pueden elaborar discursos rigurosos que demuestren la inclusión de reflexiones propias sobre la actividad proyectual y expliciten las razones por las cuales un proceso de diseño arroja un resultado preciso.

Adicionalmente, se busca acercar a los estudiantes a diferentes disciplinas para que junto con ellas puedan proponer soluciones integrales a problemas reales y así complementar los procesos de planificación y modelación de los proyectos desde el diseño, para optimizar recursos y obtener mejores resultados.

También, se propone desde el LEDI articular el pensamiento en diseño en el contexto académico de los otros programas de la Universidad de Caldas, con 
el fin de extender este alcance al trabajo con entidades sin ánimo de lucro y motivar la participación en la sociedad de los estudiantes de Diseño Visual, a partir de la confrontación de saberes teóricos en la práctica.

\section{Conclusiones}

El principal interés de un docente universitario en cualquier área del conocimiento debe ser aportar a la formación integral de sus estudiantes. Complementario a lo anterior, en la enseñanza del diseño hay un reto adicional, tratándose de una disciplina en constante cambio y que impacta de una forma contundente diversas actividades humanas indispensables para la vida, como lo son la comunicación, la habitabilidad, la interacción tecnológica y en general para habitar en un mundo que requiere mediaciones entre las personas y con los objetos y espacios.

La enseñanza del diseño como actividad práctica, permite descubrir los modos de racionalizar su acción en determinados escenarios, esto permite su observación, reflexión y análisis, y a su vez una visión teórica que sustente el ejercicio práctico.

El proyecto de diseño le permite al docente planificar las secuencias didácticas y mediar procesos entre instituciones y estudiantes; al estar la práctica pedagógica en el centro de la actividad docente es válido que los profesores de diseño implementen recursos disciplinares para inducir el trabajo en clase. En esta experiencia educativa presentada se plantea el potencial del proyecto de diseño como recurso didáctico de dominio propio de la disciplina, que opera como mediador entre las didácticas que incentivan las habilidades y los contenidos que afianzan las competencias de los estudiantes en el aula (Figura 6). 


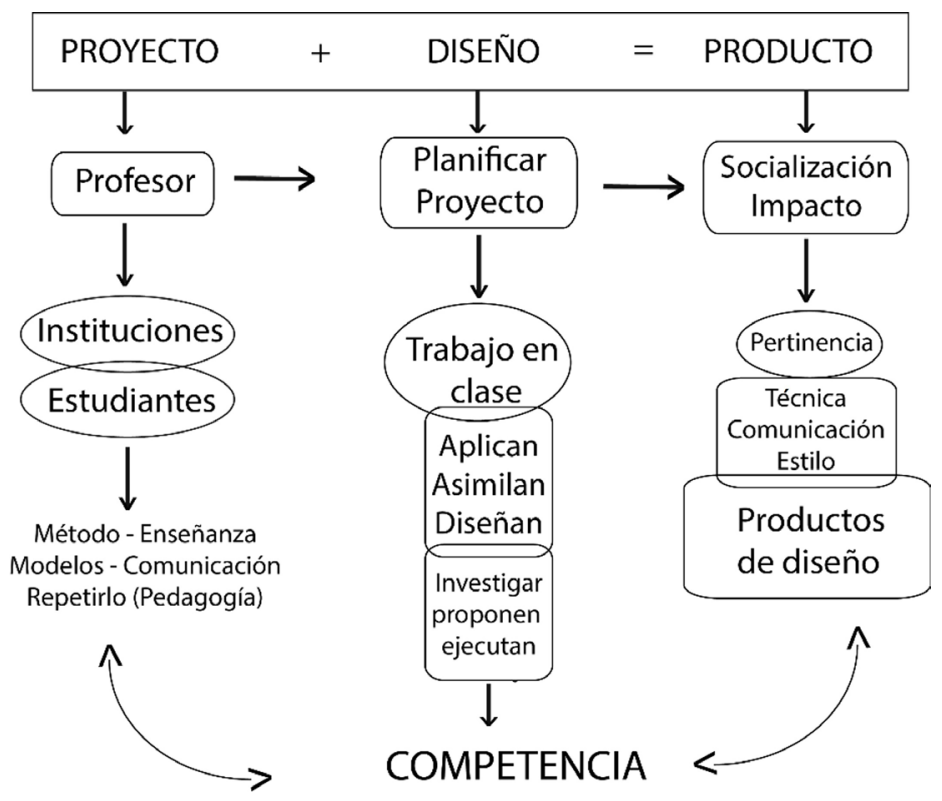

Figura 6. Síntesis de la ruta del proyecto de diseño. Fuente: elaboración propia.

El objetivo de la formación no debe ser solo instrumental, debe corresponder a un cambio conceptual profundo y a una postura reflexiva y propositiva que deben estar presentes en los productos que plantean en el acto de diseño; al tener una experiencia práctica real el aprendizaje, será validado en una esfera que al estudiante lo puede motivar e involucrar más en su proceso de apropiación del conocimiento.

La multiplicidad de teorías en diseño y sus diversas interpretaciones por las diferentes comunidades académicas, así como el amplio espectro de desarrollos metodológicos inherentes a los procesos conducentes a la 
solución de problemas contextualizados, se complementa con la variedad de perspectivas didácticas empleadas para la formación de futuros profesionales. Todo esto lleva a plantear nuevos retos formativos y didácticos orientados a perfilar soluciones propias de la disciplina; por esto, se propone como una alternativa complementaria a la formación profesional en diseño el uso de proyectos en laboratorios especializados.

En el campo disciplinar, el diseño ha planteado y superado retos humanos, unos con mayor impacto que otros, dentro de los contextos económicos con su idea de progreso para democratizar el uso de productos y servicios, dando una idea de bienestar social a veces equivocada. Es por esto por lo que urge dar una mirada a las prácticas docentes de dominio específico de diseño que contribuyan a la formación de diseñadores, no solamente competentes y hábiles desde las destrezas técnicas del oficio, sino sobre todo profesionales propositivos, informados, conscientes de su contexto y a la altura de los desafíos de un mundo cada vez más caótico, saturado, contaminado e intervenido, en algunas ocasiones por la misma disciplina del diseño.

\section{Referencias}

Alexander, C. (1980). Tres aspectos de matemáticas y diseño. Tusquet Editores.

Ambrose, G. y Harris, P. (2010). Metodología del diseño. Parramón.

Archer, L. B. (1964). Systematic Methods for Designer. Council of Industrial Design.

Asimov, M. (1970). Introducción al proyecto. Editorial Herrero Hnos. Sucs.

Brown, T. (2009). Change by Design: how Design Thinking Transforms Organizations and Inspire Innovation. Harper Business.

Chaves, N. (1988). La imagen corporativa. Teoría y metodología de la identificación institucional. Gustavo Gili. 
Cloninger, C. (2007). Hot-wiring your creative process. Strategies for print \& media designers. New Riders.

Creswell, J. (1998). Qualitative inquiry and research design. Choosing among five traditions. Sage.

Cross, N. (2012). Métodos de Diseño. Estrategias para el Diseño de Productos. Limusa Wiley.

Davis, M. (2016). Normal Science' and the Changing Practices of Design and Design Education. Visible Language, 50(1).

Díaz, G., Mendoza, Y. y Escorcia, S. (2021). Transposición didáctica y la perspectiva crítica de Pablo Freire. Encuentros. Revista de Ciencias Humanas, Teoría Social y Pensamiento Crítico, 1(13), 168-176.

Frascara, J. (2006). El diseño de comunicación. Edición corregida y extendida de Diseño Gráfico y Comunicación. Ediciones Infinito.

Fundación Nutrir. (2021). ¿Quiénes somos? https://nutrirong.com/quienes-somos/

González, E. (1994). El proceso de creación y la evolución de los proyectos de diseño gráfico. Servicio Editorial Universidad del País Vasco.

Hernández, R., Fernández, C. y Baptista, P. (2014). Metodología de la investigación (6a. Ed.). McGraw-Hill.

Jones, J. C. (1976). Métodos de diseño. Gustavo Gili.

Lauer, D. A. (1979). Design Basics. Holt, Rinehart and Winston.

Lave, J., \& Wenger, E. (1991). Situated Learning: Legitimate Peripheral Participation. Cambridge University Press.

Lawson, B. (2005). How Designers Think. The Design Process Demystified. Architectural Press.

Loaiza, Y. (2019). Las prácticas pedagógicas en el campo de la educación: su confluencia en investigaciones de posgrado de la UCM. Revista de Investigaciones UCM, 19(33), 49-63.

Londoño, F. (2014). Plan de Acción Institucional de la Universidad de Caldas, "Universidad y Pensamiento Crítico 2014-2018". Universidad de Caldas. http://www.ucaldas.edu.co/ portal/wp-content/uploads/2014/12/PAI_Plan_Accion_Institucional_Rectoria_2014_2018. pdf 
López, A. M. y Tamayo, Ó. E. (2012). Las Prácticas de laboratorio en la enseñanza de las ciencias naturales. Revista Latinoamericana de Estudios Educativos, 8(1), 145-166.

Moles, A. y Caude, R. (1977). Creatividad y métodos de innovación. Ibérico Europea de Ediciones.

Mollerup, P. (1987). El programa de diseño corporativo. IMPI/MINER Ediciones de Diseño. Fundación BCD Barcelona.

Munari, B. (1981). ¿Cómo nacen los objetos? Apuntes para una metodología proyectual. Gustavo Gili.

Ortega-Díaz, C. y Hernández-Pérez, A. (2015). Hacia el aprendizaje profundo en la reflexión de la práctica docente. Ra Ximhai, 11(4), 213-220.

Plan Institucional de Actividad Académica. (2015). PIAA. Diseño Integral.

Radio Nacional de Colombia. (2014). Visual Rock: proyección audiovisual para las bandas manizaleñas. https://www.radionacional.co/noticia/visual-rock-proyeccion-audiovisualpara-las-bandas-manizalenas

Ricard, A. (2008). Conversando con estudiantes de diseño. Gustavo Gili.

Rodríguez, L. (2006). Diseño: estrategia y táctica. Siglo XXI.

Rodríguez, L. (2011). Interdisciplina en diseño: un reto para la docencia. Diseño sin fronteras. IV Congreso Internacional de Diseño. México 26-28 de octubre 2011. http://www.dis.uia.mx/ conference/2011/LibroMX2011.pdf

Tobón, S., Pimienta, J. y García, J. (2010). Secuencias Didácticas: aprendizaje y evaluación de competencias. Pearson-Prentice Hall.

Wenger, E. (1998). Communities of Practice: learning, meaning and identity. Cambridge University Press.

Zabala, A. (2000). La práctica educativa. Cómo enseñar. Graó.

Cómo citar: Castaño, J., Tamayo, Ó. E. y Castañeda, W. (2022). Un escenario para el aprendizaje a través de proyectos de diseño en contextos sociales: Laboratorio Estratégico de Diseño Integral (LEDI). Revista KEPES, 19(25), 135-164. https://doi.org/10.17151/kepes.2022.19.25.6 